\title{
Imagen corporal y participación en las clases de Educación Física Body image and participation in Physical Education classes
}

*Roberto Lagos Hernández, *Tatiana Jerez Fuenzalida, **Gerardo Fuentes-Vilugrón, *Adolfo Willschrei Barra, *Karen Acuña Molina, *Macarena Leichlte San Martín

*Universidad Autónoma de Chile (Chile), **Universidad Católica de Temuco (Chile)

\begin{abstract}
Resumen. Esta investigación tiene por objetivo estudiar la incidencia de la imagen corporal en la participación en clases de Educación Física de 45 damas y varones de entre 13 y 17 años de establecimientos educacionales de Temuco, Chile. Se consideró aquellos alumnos que, sin tener impedimento de salud, no participaban regularmente de la clase de Educación Física. La metodología contempló un enfoque mixto, con un diseño no experimental del tipo descriptivo transeccional. Se hizo una recolección sistemática de antecedentes cualitativos a través de grupos focales y se consideró el Body Shape Questionnaire, para estudiar la opinión de los estudiantes respecto de su imagen corporal. Los resultados muestran que un 10\% de los varones manifiestan preocupación leve respecto de su imagen corporal y que un $24 \%$ de las damas manifiestan una preocupación moderada respecto de su figura. Estos datos se corresponden con las opiniones manifestadas en los grupos focales, donde se pudo apreciar que los estudiantes sienten incomodidad e insatisfacción corporal en relación con su cuerpo, especialmente el género femenino. Sin embargo, esto no se constituye como la razón principal para no participar de las clases de Educación Física, siendo aspectos metodológicos los que son mencionados como de mayor importancia.
\end{abstract}

Palabras clave: imagen corporal, educación física, participación escolar.

Abstract. The aim of this research is to study the incidence of body image in the participation in Physical Education classes of 45 girls and boys between 13 and 17 years old from educational establishments in Temuco, Chile. We considered those students who, without having a health impediment, did not participate regularly in Physical Education classes. The methodology contemplated a mixed approach, with a non-experimental design of the transectional descriptive type. A systematic collection of qualitative data was made through focus groups and the Body Shape Questionnaire was used to study the students' opinion of their body image. The results show that $10 \%$ of the males expressed mild concern about their body image and $24 \%$ of the females expressed moderate concern about their body shape. These data correspond to the opinions expressed in the focus groups, where it could be seen that students feel discomfort and body dissatisfaction in relation to their body, especially the female gender. However, this does not constitute as the main reason for not participating in Physical Education classes, being methodological aspects those that are mentioned as of greater importance.

Key words: body image, physical education, school participation.

\section{Introducción}

La preocupación por la incidencia de la imagen corporal en relación con la participación de adolescentes en prácticas de actividad física ha sido tema de interés (Vaquero-Cristóbal et al., 2013; Colunga-Rodríguez et al., 2020), donde se ha podido constatar la vinculación entre ambos factores (Ceballos-Gurrola et al., 2020; Reyes, Delgado-Floody, \& Martínez-Salazar, 2020), con resultados contradictorios, en algunos casos se señala que una pobre percepción de la imagen podría incrementar la actividad física por efectos de una mejor autoestima o necesidad de figuración y aceptación social, pero otros trabajos describen efectos contrarios (dos Santos, \& Leal, 2017), donde la obesidad, la falta de

Fecha recepción: 10-09-21. Fecha de aceptación: 19-11-21

Gerardo Fuentes-Vilugrón

gfuentes@educa.uct.cl autoeficacia, la torpeza motriz o bien la falta de autoestima, reducirían el interés por al actividad motriz (Blanco et al., 2016), por lo tanto, profundizar en las relaciones entre imagen corporal y participación en clases de Educación Física constituyen una contribución para entender cómo se desarrolla este fenómeno en las aulas donde no se deben además, dejar de lado aspectos culturales y étnicos (Jansen, Zayed, \& Kittsteiner, 2020). Para tal propósito en esta investigación se abordan tres aspectos, la falta de interés por la Educación Física de los estudiantes de educación media, la percepción de la imagen corporal que manifiestan respecto de sí mismos y la potencial incidencia de ambos factores en el desinterés por la clase de Educación Física. Se ha descrito durante los últimos años una apatía por las clases de Educación Física lo que ha motivado una mayor preocupación en los procesos educativos relacionados con la Educación Física de los estudiantes chilenos (Moreno, Gamboa, \& Poblete, 2014), hecho que se ha materiali- 
zado en la implementación de diversas estrategias educativas que tienen como propósito lograr la participación de sus estudiantes y mejorar la calidad y equidad de los procesos formativos. Esto, se constituye en una acción determinante, considerando el actual escenario de sedentarismo presente en nuestro país (Osses \& Jaramillo, 2008; Serón, Muñoz \& Lanas, 2010), lo que se ha traducido en importantes esfuerzos por transformar a las comunidades educativas en establecimientos promotores de salud, acciones que se han desarrollado en conjunto con estamentos gubernamentales desde inicios del año 2000 a la fecha (Salinas \& Vio, 2002). El fenómeno de la adherencia a la actividad física es un tema que se han investigado internacionalmente desde hace varios años (Galán et al., 2014; Malina, 2012), pero no hay abundante información en el contexto chileno, salvo algunas investigaciones que han abordado los motivos por la práctica de actividad física en la clase de Educación Física (Peña-Troncoso et al., 2021)

En esta línea de acción, el subsector de Educación Física en Chile busca la formación integral de los estudiantes y a partir de la actividad física, lograr desarrollar habilidades motrices, actitudes proclives al juego limpio, el liderazgo y el auto cuidado, dando posibilidad de adquirir un estilo de vida activo y saludable, asociados a beneficios tanto individuales como también sociales. (Ministerio de Educación, 2013; Cádiz et al., 2021). Estas orientaciones ministeriales, tienen por misión ofrecer una educación que se adapte a las necesidades de la infancia y la adolescencia, periodo de la vida marcada por gran vulnerabilidad, debido a que representan etapas en las que el ser humano crece y se desarrolla, tanto física como intelectualmente (Fuentes-Vilugron \& Lagos-Hernández, 2020; Miranda et al., 2014).

La Educación Física como disciplina pedagógica busca la formación intelectual y motriz de sus estudiantes, sin embargo, es necesario profundizar en el estudio de los aspectos motivacionales que impulsan a los estudiantes, para participar activamente de esta actividad, constituyéndose esta tarea en una acción de complejidad, ya que son muchos los elementos que inciden en la percepción respecto de la Educación Física y la motivación de los estudiantes por participar de sus clases regulares (Cuadra, Georgudis, \& Alfaro, 2012; Fuentes \& Lagos, 2019). Así, la falta de motivación por asistir y participar en las clases de Educación Física se constituiría en uno más de los factores que colabora negativamente en la situación de sedentarismo que viven los escolares chilenos, especialmente en la educación media. Habiéndose observado durante los últimos años, que este grupo es el que más ha aumentado en cuanto a inactividad física (Aguilar-Farias et al., 2020), sumando además alteraciones en la conducta alimentaria (Rodriguez \& Pizarro, 2006; Lizana et al., 2011).

No hay cifras oficiales que establezcan la prevalencia de rechazo a la clase de Educación Física en Chile, sin embargo, se tiene la apreciación de que, en este periodo, ésta disciplina pedagógica, adquiere un papel importante en la promoción de conductas que mejoran la calidad de vida (Pérez \& Delgado, 2009; Stuart, Lopez \& Granado, 2012). Por eso, se estima relevante, establecer las causas que manifiestan los estudiantes para rehusarse a participar de las prácticas propias de este subsector.

Otros de los elementos considerados en este estudio, es la percepción que tienen los estudiantes respecto de su imagen corporal. Se ha podido constatar que la percepción de la imagen corporal es uno de aquellos elementos que influye decididamente en que las personas tengan una actitud más activa frente al movimiento (Fernandez et al., 2010; Estévez, 2012; Ornelas et al., 2020). No obstante, muchos adolescentes, más damas que varones, aprenden a odiar la Educación Física, aprenden a sentirse incompetentes motrices, a tener autoconceptos bajos y/o negativos sobre su capacidad físico motriz y su corporalidad (López, 2012). Además, los avances tecnologicos de la actualidad, hacen cada vez menos necesario utilizar el movimiento, una razón más para el aumento del sedentarismo y enfermedades en los adolescentes (Cárcamo, 2012) en un periodo crítico en el desarrollo humano, en cuanto a comportamientos y estilos de vida saludables (Newman, et al., 2008; Requena, 2012) y no tan solo en el ámbito de las conductas alimentarias o de actividad motriz, sino también, en la formación y maduración de la imagen corporal, ya que a esta edad, se producen una serie de cambios que provocan una mayor preocupación por parte de los jóvenes hacia su apariencia (Ramos, Rivera, \& Moreno, 2010). Cabe destacar que la imagen corporal se desarrolla paralelamente a la identidad del individuo en interaccion entre factores personales, psicológicos, sociales y emocionales y a esto se le agrega una mezcla entre emociones, intenciones, aspiraciones y tendencias personales (Cordeiro et al., 2012).

La imagen corporal es un constructo teórico relacionado con la percepción de la propia imagen y se le relaciona a la identidad, los sentimientos y las conductas (Pérez \& Romero, 2010). Su estudio tiene especial importancia por las relaciones con actitudes y conductas asociadas al logro de un cuerpo socialmente acepta- 
ble (Perpiñá, 1987; Wardle, 1988), aspecto que se considera clave en la constitución de la imagen corporal (Perpiñá \& Baños, 2007; Behar, 2010; Trejo et al., 2010). Otros acercamientos al concepto imagen corporal la consideran como auto concepto físico, vinculado a la apariencia física, la condición física y la sexualidad (Woodburn, Boschini, \& Fernández, 1997) siendo un proceso en constante ajuste que sintetiza el desarrollo del sujeto y su identidad (Aguado Vasquez, 2004; Alacid et al., 2013)

Como se mencionó precedentemente, la génesis de la presente investigación vincula el abandono de la clase de Educación Física y la percepción de la propia imagen, por tanto, es de interés de estos investigadores, estudiar la incidencia de la imagen corporal en la participación en clases de Educación Física de 45 damas y varones, estudiantes de educación media, de entre 13 y 17 años pertenecientes a 5 establecimientos educacionales de la ciudad de Temuco, Chile.

\section{Metodología}

La metodología contempló un enfoque mixto, con un diseño no experimental del tipo descriptivo transeccional (Hernández, Fernández \& Baptista, 2014).

\section{Participantes}

La muestra fue no probabilística e intencionada. Respecto del criterio de inclusión de los participantes, se consideró a todo estudiante de entre 13 años 0 días y 17 años 364 días de 5 establecimientos municipales, que manifestaran voluntariamente no participar de manera regular de la clase de Educación Física y que comunicara oficialmente su decisión al docente del subsector, quien registró su nombre y lo hizo llegar a los investigadores para incluirlo en la muestra con la autorización del estudiante o su tutor legal. En total se pesquisaron un total de 45 estudiantes de un total de 900, que reunían las condiciones de los criterios de inclusión, representando un $5 \%$ de total.

\section{Instrumentos}

Para efectos de esta investigación fueron utilizados dos instrumentos de recolección de datos: el Body Shape Questionnaire (BSQ) que consiste en un cuestionario sobre forma y percepción corporal, diseñado por Cooper y Taylor (1987). Este instrumento consta de 34 ítems referentes a la autoimagen, que se evalúan mediante una escala de frecuencia de 5 puntos para uno de los reactivos donde $1=$ nunca, $2=$ casi nunca; $3=$ a veces,
$4={ }_{\text {casi siempre y } 5}=$ siempre. A partir de la puntuación total se establecen cuatro categorías o niveles de preocupación por la imagen corporal: a) no hay preocupación de la imagen corporal (puntuación $<80$ ), b) Leve preocupación (puntuación entre 81 y 110), c) Preocupación moderada (puntuación entre 111 y 140) y d) Preocupación extrema (puntuación >141 puntos).

En relación con la confiabilidad y validez del BSQ, se señala que la consistencia interna se calculó por medio de Alfa de Cronbach siendo considerada alta, con coeficiente de correlación intraclase cuya medida fue de 0,95 para el primer factor y 0,92 para el segundo factor, presentando un alto nivel de homogeneidad y consistencia interna.

El otro instrumento utilizado es un Grupo Focal, este método valora la interacción y estimula el dialogo sobre asuntos que podrían ser complejos de abordar y tiende a profundizar en las informaciones y entender comportamientos en un determinado contexto cultural (Buss et al., 2013). Los grupos focales son entendidos como una forma de recolectar datos cualitativos, donde, esencialmente, implica involucrar a un pequeño conjunto de personas en una discusión de grupo informal, enfocada en un tema específico (Onwuegbuzie et al., 2011).

Para los grupos focales, se utilizaron 7 reactivos aprobados por criterio de Delphi, donde se hizo énfasis en la validación de contenido con el propósito de asegurar reactivos coherentes en cuanto a las dimensiones de la investigación y que contaran con los atributos, fiabilidad y adecuada relación teórica (Ruiz Bueno, 2014; Escobar-Pérez \& Cuervo-Martínez, 2008; Argibay, 2006). Finalmente se aceptaron un total de 7 reactivos con participación de un total de 5 expertos, todos con experiencia en investigación y publicaciones indexadas. El procesimiento implicó con tres etapas de correcciones hasta llegar a la versión final corregida (Astigarraga, 2003) que contó con una etapa de presentación de antecedentes, una etapa de revisión y corrección según los comentarios de los investigadores revisores y finalmente la etapa donde se generó la versión final con las adecuaciones finales (George \& Trujillo, 2018). Los reactivos fueron probados en una versión piloto y son los que se presentan a continuación:

\section{Reactivos}

1. ¿Qué opinan respecto de las clases de educación física?

2. ¿Conocen los beneficios que les proporciona participar en las clases de educación física?

3. ¿Cuáles son a su juicio los motivos principales 
para no participar en las clases de Educación Física?

4. ¿Qué entienden por imagen corporal?

5. ¿Estás conforme con tu cuerpo?Y si no es así,

6. ¿Cómo te gustaría ser?

7. ¿Cómo quién te gustaría ser?

A partir de estas preguntas los participantes de los grupos focales emitieron sus respuestas, teniendo el equipo entrevistador la especial preocupación, por no direccionar, ni intencionar ninguna de ellas. Fueron incluidas en la discusión de esta investigación, todas aquellas respuestas que se reiteraron en al interior de cada grupo focal por establecimiento, siendo agrupadas en 18 afirmaciones finales. (Onwuegbuzie et al., 2011).

\section{Procedimiento para aplicación de cuestionario}

Para efectos de la aplicación del cuestionario, en esta investigación los grupos se constituyeron con un mínimo de 6 y un máximo de 8 estudiantes, en particular, 5 grupos focales con 6 estudiantes, un grupo focal con 7 estudiantes y un último grupo focal con 8 estudiantes, en todos hubo participación tanto de damas como varones. Las entrevistas las dirigió un moderador entrenado en el propio establecimiento y en horario de la clase regular de Educación Física. Se solicitó una sala regular de clases y se organizó el espacio de tal manera de formar un semicírculo con el moderador en el centro. En todo momento el moderador estuvo acompañado de un asistente, cuya función fue apoyar en el registro y toma de antecedentes. Cada grupo focal se desarrolló en un mínimo de 35 y un máximo de 60 minutos. La función del moderador era orientar las preguntas relacionadas con los dos ejes temáticos, Imagen corporal y Educación Física. Antes de iniciar los grupos focales y con la necesidad de establecer un adecuado funcionamiento de los mismos, se hizo una prueba piloto con estudiantes de Pedagogía en Educación Física de primer año de universidad, en condiciones y tiempos similares a las que se materializarían más tarde en los colegios, apreciándose que tanto el moderador y asistente, podían manejar la actividad con fluidez, objetividad y especialmente, no generando diálogos inductores de respuestas artificiales por parte de los participantes. Aprobado el piloto se iniciaron los grupos focales, siguiendo el protocolo establecido (Onwuegbuzie et al., 2011).

Todas las respuestas fueron grabadas en un dispositivo digital Marca Sony $®$ ICD-PX440 y posteriormente los antecedentes fueron trascritos literalmente en procesador de textos Windows Word (C) 2016, posteriormente las transcripciones fueron procesadas a través del Sofware Atlas-tiC, cuyo objetivo es el análisis de datos textuales y su organización en árboles de palabras y conceptos claves.

\section{Análisis de los datos}

Respecto al proceso de análisis de la información cualitativa se estructuró una codificación abierta donde a partir de sus dimensiones y significados se creó una categorización preliminar con conceptos generales (Flick, 2004) que posteriormente sirvieron de base para la elaboración de una codificación axial con la cual se gestionaron las categorías obtenidas en la codificación abierta y sus subcategorías. Se debe tener en consideración que una categoría representa un fenómeno significativo para los entrevistados. Se procedió a la codificación teórica con el propósito de obtener una categoría central que exprese el fenómeno de investigación e integre las categorías y subcategorías de la codificación abierta y axial (Strauss \& Corbin, 2002), finalmente la estructuración de mapas de análisis se llevó a cabo con software Atlas tiC, versión 8.0, desde dónde se elaboraron las discusiones y análisis conceptuales.

Los datos cuantitativos incorporaron análisis de frecuencias y porcentajes y en relación con los datos y puntuaciones del BSQ se categorizaron los niveles de preocupación según lo señalado por Cooper y Taylor (1987).

\section{Consideraciones éticas}

La presente investigación contó con el consentimiento y asentimiento informados de todos los padres y apoderados de los participantes, estudiantes y directores de establecimientos, quienes autorizaron el uso de la información con fines científicos. Por último, se deja constancia que este trabajo respetó los postulados de la declaración de Helsinki y sus actualizaciones al año 2015, para afectos de las investigaciones con seres humanos. (Asociación Médica Mundial, 2015).

\section{Resultados}

La tabla 1 muestra la distribución porcentual de los estudiantes según el establecimiento de origen, todos sin excepción, rehúsan sistemáticamente a participar de las clases de Educación Física y presentaron estos antecedentes de manera oficial para no ser parte de la

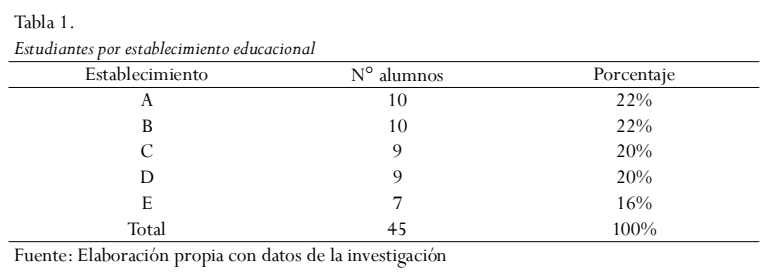


asignatura. De los sujetos estudiados, 34 personas son de género femenino y 11 de género masculino, todos pertenecientes a la comuna de Temuco. (Tabla 1).

La tabla 2 presenta el diagnóstico observado en cuanto a la imagen corporal, pudiéndose apreciar que del total de participantes, el 58\%, correspondiente a 26 sujetos evaluados, manifiestan no tener preocupación por su imagen corporal, 24\% equivalente a 11 participantes, manifiestan preocupación leve, el 18\%, es decir, 8 evaluados, muestran preocupación moderada y por último, no se observan sujetos con preocupación extrema.

\begin{tabular}{lcc} 
Tabla 2. & & \\
Diagnóstico Global BSQ & $\mathrm{N}^{\circ}$ & $\%$ \\
\hline Nivel de preocupación & 0 & 0 \\
\hline Preocupación extrema & 8 & 18 \\
Preocupación Moderada & 11 & 24 \\
Preocupación Leve & 26 & 58 \\
\hline Sin Preocupación &
\end{tabular}

En relación con el género y el diagnóstico arrojado por el BSQ, la tabla 3 presenta estos antecedentes, mostrando los resultados tanto de damas como varones. Se puede apreciar que el total de evaluados de sexo masculino suman total de 11 personas, de los cuales solo $1 \mathrm{de}$ ellos, manifestó preocupación por su imagen corporal, con un diagnóstico leve. No hubo casos observados de género masculino, con preocupación moderada ni preocupaTabla 4.

Aabla 4. Afirmaciones por establecimient

\begin{tabular}{|c|c|c|}
\hline \multicolumn{2}{|c|}{ Establecimiento Dimensión Educación Física } & Dimensión Imagen Corporal \\
\hline \multirow[t]{5}{*}{ A } & "la mayoría de sus clases de Educación Física son repetitivas & " "se sienten incómodas con su cuerpo, produciendo \\
\hline & "hacen diferencia entre hombres y mujeres" & desconfianza" \\
\hline & "sienten que no les beneficia en nada la clase". & \\
\hline & "falta motivación por parte del profesor" & "les gustaría cambiar su cuerpo" \\
\hline & $\begin{array}{l}\text { "estudiantes no poseen noción acerca de los beneficios de la } \\
\text { actividad física" }\end{array}$ & \\
\hline \multirow[t]{2}{*}{ B } & $\begin{array}{l}\text { "las clases de Educación Física son aburridas, rutinarias, } \\
\text { repetitivas y falta didáctica" }\end{array}$ & "sienten insatisfechas". \\
\hline & $\begin{array}{l}\text { "no existe nada entretenido que les pueda brindar" } \\
\text { "miedo a sufrir lesiones". }\end{array}$ & \\
\hline \multirow[t]{3}{*}{$\mathrm{C}$} & $\begin{array}{l}\text { "no es relevante para ellos los objetivos de la clase de } \\
\text { educación física" }\end{array}$ & $\begin{array}{l}\text { "se preocupan por su imagen corporal" "buscan estereotipos } \\
\text { de personas ideales en las que les gustaría verse reflejadas" }\end{array}$ \\
\hline & "hay poca motivación por parte del profesor" & \\
\hline & "los horarios de la clase son mal establecidos" & \\
\hline \multirow[t]{3}{*}{$\mathrm{D}$} & "sienten desmotivados" & "no se sienten conformes con su propio cuerpo" \\
\hline & "no les gusta el método en que el docente realiza las clases" & $\begin{array}{l}\text { "su prototipo a seguir se relaciona a los medios de } \\
\text { comunicación" }\end{array}$ \\
\hline & & "que les gustaría ser modelos o cantantes". \\
\hline E & $\begin{array}{l}\text { "Se sienten desmotivados ya que no les gusta el método en } \\
\text { que el docente realiza las clases" }\end{array}$ & \\
\hline
\end{tabular}

ron ningún comentario favorable respecto de la asignatura, lo que se puede comprender, considerando que todos han rehusado participar de las clases del subsector. Considerando la codificación preliminar, se clasificaron las respuestas en afirmaciones, organizándolas según su procedencia por establecimiento. En el caso de respuestas que se repitieron, se le atribuyó la procedencia al establecimiento en donde se emitió por primera vez la afirmación. En general, las respuestas tenían muchas similitudes sin embargo hubo un total de 18 afirmaciones puras que fueron distribuidas según su origen en cada uno de los establecimientos. Se procedió a clasificar las opiniones con mayor presencia por establecimiento, pudiéndose apreciar en la Tabla número 4, el resultado de esta organización.

En cuanto a los resultados de los grupos focales por establecimiento, los estudiantes del colegio «A» coinciden en que «la mayoría de sus clases de Educación Física son ción extrema. De las 34 damas eva-

luadas, un 53\% correspondientes a 24 personas, manifiestan preocupación moderada por su imagen corporal y 10 con un 22\% manifiestan preocupación leve, en relación a los varones 10 señalan tener una preocupación leve, representando esto un $24 \%$. No se aprecian casos de estudiantes del género femenino con preocupación extrema dentro de los sujetos evaluados.

\begin{tabular}{|c|c|c|c|c|}
\hline \multicolumn{5}{|c|}{ orporal según Sexo } \\
\hline Nivel de preocupación & $\mathrm{N}^{\circ}$ Damas & $\%$ & $\mathrm{~N}^{\circ}$ Varones & $\%$ \\
\hline Preocupación Moderada & 24 & 53 & 0 & 0 \\
\hline Preocupación Leve & 10 & 22 & 1 & 2 \\
\hline
\end{tabular}

\section{Resultados grupos focales}

Los resultados derivados de la aplicación de los grupos focales, en donde se hizo una codificación preliminar de las afirmaciones, categorizándolas respecto de su relación con la Educación Física y el concepto imagen corporal, logró evidenciar que los estudiantes no hicie- repetitivas», los profesores «hacen diferencia entre hombres y mujeres» y que «sienten que no les beneficia en nada la clase». Sobre imagen corporal, en especial las damas «se sienten incómodas con su cuerpo, produciendo desconfianza».

En cuanto a los estudiantes del establecimiento educacional «B» señalan que «les falta motivación por parte del profeson» y que los estudiantes «no poseen noción acerca de los beneficios de la actividad física». La mayoría de los estudiantes hace alusión a que «les gustaría cambiar su cuerpo», especialmente el género femenino. En el establecimiento educacional «C» los estudiantes mencionan que «las clases de Educación Física son aburridas, rutinarias, repetitivas y falta didáctica», «no existiendo nada entretenido que les pueda brindar», además manifiestan «miedo a sufrir lesiones». La mayoría de las alumnas sobre imagen corporal se «sienten insatisfechas». En establecimiento educacional «D» los alumnos señalan que «no es relevante para ellos los objetivos de la clase de educación física», «habiendo poca motiva- 
ción por parte del profesor», por otro lado, mencionan que «los horarios de la clase son mal establecidos». La mayoría de los estudiantes «se preocupan por su imagen corporal» $\mathrm{e}$ indican que «buscan estereotipos de personas ideales en las que les gustaría verse reflejadas» Por último en la escuela «E» los estudiantes evaluados se «sienten desmotivados», «ya que no les gusta el método en que el docente realiza las clases». Con respecto a la imagen corporal, el género femenino indica que «no se sienten conformes con su propio cuerpo», «su prototipo a seguir se relaciona a los medios de comunicación» y señalan «que les gustaría ser modelos o cantantes».

\section{Discusión}

Las investigaciones en relación con la imagen corporal y la educación física han manifestado resultados contradictorios debido a la naturaleza multifactorial que inciden en que inciden en la realización de la actividad física (Contreras et al., 2012; do Santos \& Leal, 2017). En relación con la imagen del cuerpo humano se entiende como la figuración de un cuerpo formado en la mente de una persona, mientras que la imagen corporal es multidimensional y está influenciada por factores sociales, fisiológicos, psicológicos y ambientales (Finato et al., 2013; Durán et al., 2013). Los resultados de este estudio en relación a la imagen corporal, obtenidos mediante la utilización del instrumento BSQ y grupos focales, indican que la imagen corporal es un componente que tiene interés por parte de los estudiantes situación que se ha evidenciado además en otras indagaciones (Ceballos-Gurrola et al., 2020), pero que en este caso, se considera poco incidente en la intención por participar de las clases de Educación Física, esto se afirma considerando que un 58\% de las personas evaluadas manifestaron que no hay mayor preocupación por su imagen corporal, sin embargo sí afirmaron en los grupos focales, su desinterés por ciertos elementos constitutivos de la clase. Se insiste en el rol del docente en cuanto a ser un agente de motivación permanente, ya que las vivencias específicas que suponen dichas clases dan lugar a una valoración de estas por parte del alumno. Esta valoración, en conjunto, va conformando actitudes más o menos positivas, actitudes que pueden a su vez generar hábitos de práctica de ejercicio físico (Moreno \& Cervelló, 2003). En relación a este fenómeno Sabatini (2006) señala que los jóvenes en general prefieren hacer actividad física más que otra actividad, en especial los varones, que las expectativas pasadas son determinantes para la adherencia a la práctica y la que la intervención del docente es determinante para la adherencia a la práctica de actividad física, sin embargo, la sensación de autoeficacia es muy importante para efectos de sentirse motivado por la Educación Física. El estudio permitió apreciar que, en relación con imagen corporal, se observa mayor preocupación en el género femenino, esto también se ha observado en otras investigaciones con grupos de similar edad y localización geográfica (Lagos, 2010; Salvador et al., 2018), donde los resultados declaran que el género femenino es el más sensible a esta apreciación corporal y su vínculo con la actividad física (Da Silva et al., 2008). Al comparar esta investigación con una realizada en el año 2009, donde se evaluó a estudiantes universitarios se evidenció que el género femenino tenia puntuaciones significativamente más altas en el BSQ en comparación con el género masculino (Salvador, et al., 2018), además presentaban una tendencia mayor que los hombres, a escoger figuras corporales de bajo peso, a la hora de representar las dimensiones corporales que tenían o deseaban (Nichols et al., 2009). De igual manera, según resultados obtenidos en una investigación realizada en Brasil en el año 2012, allí se identificó que las adolescentes del género femenino presentaron niveles de alteración en la imagen corporal, con alta prevalencia de insatisfacción con su propio cuerpo, lo que se asemeja a los datos de este trabajo, no por el grado de insatisfacción, sino en la comparación con respecto al género masculino (Cordeiro et al., 2012). Sin embargo y en contraste, otra investigación desarrollada en Europa entregó resultados distintos a los arrojados en este estudio, dado que, en ese caso, los estudiantes de género masculino, presentaba niveles de insatisfacción significativamente mayores que el género femenino (Gómez, 2013). A pesar de estos antecedentes donde las estudiantes de género femenino tienen mayor preocupación que los del género masculino, existe una investigación que según sus resultados indican que las alteraciones a la imagen corporal son tan frecuentes tanto en hombres como también en mujeres (Durán et al., 2013). Esta investigación no encontró casos de estudiantes con preocupación extrema en relación a la percepción de la imagen corporal lo que sería una señal positiva considerando que en el caso de los adolescentes que poseen buena percepción respecto de si mismos, tienen también mejor estado de salud y además realizan más actividad física, siendo los varones, quienes poseen mejor estado de salud y realizan más prácticas deportivas. Por el contrario, las damas, desarrollarían menos actividades motrices ya que poseen una percepción más negativa respecto de la imagen corporal (Urrutia et al., 2010). Estudios similares de estu- 
diantes sin preocupación extrema también se han encontrado en México, en particular con mujeres, lo que dejaría en evidencia que esto no es un fenómeno aislado (Valdez \& Guadarrama, 2008).

Es interesante apreciar que los antecedentes arrojados por los grupos focales dan cuenta de la disconformidad de los estudiantes respecto de su imagen corporal en solo 4 de 18 comentarios, sin embargo, esto no se ve reflejado en los resultados del test BSQ, que solo manifiesta preocupación moderada por la imagen corporal, al respecto se aprecia que hay una diferencia entre el discurso y la respuesta que dieron al ser consultados en el instrumento.

En relación con los comentarios reflejados en la presente investigación, todos sin excepción, se relacionan con opiniones no favorables respecto de la dinámica de la clase de Educación Física, también señalan desconocimiento de su valor, comentan respecto de elementos motivacionales o la capacidad profesional del docente, hecho que incide negativamente en la adherencia a la clase. Cabe señalar que, en la actividad física, los niños prefieren la diversión y hacer amigos; los adolescentes la competición y las amistades; los universitarios la aventura y la diversión; los adultos obtener actividad física regular y los adultos mayores los beneficios relacionados con la salud. Las mujeres se motivan por la apariencia y aspectos sociales, mientras los hombres por la competición y el estatus. Los sujetos que hacen deporte se motivan por la competición y los que hacen ejercicio por la imagen corporal (Azofeifa, 2006), sin embargo este fenómeno no está estudiado profundamente en lo concerniente a la clase de Educación Física, de hecho esta asignatura, que incluye elementos de deportes, actividad física y salud (Ministerio de Educación, 2013) se considera una actividad lectiva, por lo tanto está impuesta por decreto, no es libremente escogida por los estudiantes y eso podría ser una de las razones que generan rechazo escolar, más aun cuando los programas se rigen por modelos con tendencia deportivista. Hay trabajos que dan cuenta de que la Educación Física podría estar siendo víctima de una mirada muy organicista respecto del movimiento, basado en un abordaje positivista de la práctica física al interior de las unidades educativas, induciendo de esta manera a una subordinación del cuerpo-maquina y contribuyendo así a una mayor apatía de los estudiantes a la realización de actividad física regular. (Lagos, 2011)

Observados los antecedentes de este trabajo, el profesor adquiere un valor insustituible, por cuanto es su responsabilidad generar cambios en los intereses de los estudiantes. El profesor facilita el aprendizaje del alumno entregando como mínimo, la información relevante en diversas áreas del saber y éste a su vez otorga conocimiento sobre sus estrategias y motivaciones de aprendizaje. (Cea et al., 2013) Esto coincide con otras indagaciones que señalan la relevancia del rol del docente (Gutiérrez \& Pilsa, 2007, Torre, 1998;) siendo su influencia, un agente que promueve la participación activa de los alumnos en las actividades regulares del subsector (Cárcamo, 2012), en efecto, los estudiantes entregan una gran valoración a los aspectos relacionados con la personalidad del educador, esto fue estudiado en una trabajo que involucró a 1.311 escolares de $5^{\circ} \mathrm{y}$ $7^{\circ}$ año Básico y de $1^{\circ}$ y $3^{\circ}$ año Medio de establecimientos Municipales y Particulares Subvencionados pertenecientes a la Región de Araucanía, misma región donde se generó el presente trabajo. Los alumnos que perciben al profesor como democrático, alegre, justo, agradable y comprensivo, le otorgan una mayor importancia a la Educación Física. (Moreno \& Cervelló, 2003)

Las opiniones manifestadas por los estudiantes demuestran disconformidad con el rol que cumple el profesor de Educación Física, esto también fue observado en una investigación, que hacía referencia a la perspectiva que tenían los estudiantes acerca de su profesor de Educación Física, según esos resultados, se aprecian diferencias a las respuestas otorgadas por los estudiantes de este estudio, dado que, al profesor se le ve de forma positiva en cuanto a competencias y capacidades profesionales, sin embargo, se le cataloga de forma negativa en cuanto a sus métodos y a su manera autoritaria de dictar clase (Cárcamo, 2012). Asimismo, se da a conocer que las clases de Educación Física aportan considerablemente en la aceptación de la imagen corporal de los adolescentes, sin embargo en los resultados de este estudio, se puede dilucidar que la clase de Educación Física no es un factor que ayude en la mejora de la auto percepción de la imagen corporal, ya que a pesar de no participar de las clases, ninguno de los sujetos evaluados estaba en la categoría de preocupación extrema según el BSQ, lo que lleva a concluir que no hay gran incidencia de una variable sobre la otra (Onofre et al., 2009). Aparentemente, el fenómeno de falta de participación de los estudiantes en las clases de Educación Física no se debe exclusivamente en este caso, a una deficiente percepción de su imagen corporal, a esta preocupación que está presente solo de manera moderada, se suma el rol del profesor de Educación Física y su incapacidad para motivar a los estudiantes. (Moreno \& Cervelló, 2003). Pero también se puede agregar que los estudiantes de 
sexo femenino tienen tendencia a disponer una percepción negativa en relación con su imagen corporal.

Este trabajo deja en evidencia dos fenómenos, en primer lugar, cuando el docente de Educación Física desempeña su tarea educativa con calidad y eficiencia, los estudiantes satisfacen sus necesidades de movimiento, experimentando placer e inclinación hacia la práctica de actividades físicas, actitudes que se prolongarán durante toda su vida (Moreno \& Cervelló, 2003). Sin embargo, cuando las actitudes y comportamientos del profesor se alejan de lo establecido y sus prácticas son contrarias a lo esperado, los alumnos responden con indisciplina e indiferencia hacia la clase, lo que repercute en su poca inclinación hacia la actividad física escolar y en general, hacia el ejercicio físico durante la etapa de madurez (Aguayo, 2010), un segundo fenómeno da cuenta de que la imagen corporal representa una preocupación real entre entre los jóvenes (Urrutia, Azpillaga, \& Gurutze, 2010; Alacid et al., 2013) y que se han apreciado relaciones entre la inactividad física y las percepciones de la imagen corporal (Pieron, 2007; CasillasEstrella et al., 2006). Sin embargo, no siempre hay una relación directa entre ambas situaciones, ejemplo de ello, es el trabajo de Duncan et al., (2004) quienes constataron en un estudio con 277 estudiantes británicos de entre 11 a 14 años, que no hay relaciones significativas de la imagen corporal y la actividad física. No obstante, hay que salvaguardar las diferencias entre la edad y el origen de las poblaciones estudiadas. Por otro lado, en una investigación de Rodríguez, Goñi, \& Ruiz (2006) con un total de 539 estudiantes de entre 12 y 23 años, se señala que se podría atribuir una relación de causalidad entre un bajo autoconcepto personal y una baja en la condición y nivel de actividad física del sujeto y que esta situación probablemente provenga de la poca actividad que el sujeto despliega, así también, aquel que se siente físicamente incompetente, tiende a rehuir actividades físicas, sin embargo señalan los autores, no se conoce con precisión cuál es la orientación de la causalidad, aunque se cree que hay una naturaleza bidireccional en ambos fenómenos.

\section{Conclusiones}

Se puede concluir que cuando el docente de la disciplina cumple con calidad y eficiencia las actividades académicas, los estudiantes satisfacen sus necesidades y expectativas de movimiento, apreciándose mayor inclinación hacia la práctica de actividades físicas y actitudes positivas hacia el ejercicio, además los estudiantes res- ponden disciplinadamente y con atención hacia la clase. Se pudo apreciar además que no se puede atribuir una relación causal entre la imagen corporal y su incidencia en la participación de los estudiantes en las clases de Educación Física, no obstante los jóvenes efectivamente manifiestan una preocupación real por su figura y sus formas corporales, situación que se podría atribuir a una relación entre una baja autoestima personal y una carente capacidad de autorrealización motriz, lo que impacta en la condición y nivel de actividad física de los sujetos.

También se puede concluir que aquellos estudiantes que se sienten físicamente incompetentes tienden a participar menos de las clases de Educación Física. Los varones realizan más prácticas deportivas y por el contrario las damas, desarrollarían menos actividades motrices ya que poseen una percepción más negativa respecto de su imagen corporal, sin embargo, el estudio presentó una paradoja, ya que los estudiantes emitieron comentarios negativos respecto de su imagen corporal, pero esto no se vio reflejado en los resultados del test BSQ, no registrándose casos de preocupación extrema. Los alumnos del estudio criticaron la clase de Educación Física, hecho que incide negativamente en la adherencia a otras prácticas motoras, sin embargo esto se explica, ya que el total de los estudiantes de la muestra rehusó voluntariamente a participar de esta actividad académica, la que por su naturaleza de asignatura obligatoria, genera rechazo por parte de los estudiantes, sin embargo cuando los docentes facilitan el aprendizaje con información relevante, aumenta la motivación por esta disciplina.

Por último, el fenómeno de negación a participar activamente de las clases de Educación Física no se debería exclusivamente a una deficiente percepción de la imagen corporal de los estudiantes, sino que se conjugan la falta de recursos pedagógicos del docente con una insuficiente capacidad de percepción positiva de la imagen corporal de los adolescentes.

\section{Limitaciones y sugerencias}

El presente trabajo tiene como limitación el que se estudió a una población reducida de sujetos, sin embargo, estos representan efectivamente al total de escolares que rehusaron a participar de las clases de Educación Física en los establecimientos señalados precedentemente. Además, también se considera una limitación el hecho que los estudiantes sean poco profundos y reflexivos en sus respuestas, siendo reiterativos y poco 
extensos en sus opiniones. Se sugiere para futuras investigaciones, contemplar un grupo de estudiantes más amplio, aumentar el rango de edad y generar un acercamiento previo para distender aún más el ambiente previo a la realización del grupo focal, además se debe considerar una entrevista al profesional para conocer sus apreciaciones respecto de este fenómeno.

\section{Referencias}

Azofeifa, E. G. (Julio de 2006). Motivos de participación y satisfacción en la actividad física, el ejercicio físico y el deporte. (M. C. Humano., Ed.) Revistas en ciencias del movimiento humano y salud, 3(1), 1-16. Recuperado el 2015 de Diciembre de 10, de http:/ / www.revistas.una.ac.cr/ index.php/mhsalud/article/view/318

Aguado Vasquez, J. C. (2004). Cuerpo humano e Imagen Corporal. México: Instituto de investigaciones Antropológicas.

Aguilar Farias, N., Miranda-Márquez, S., Martino-Fuentealba, P., Sadarangani, K., Chandia-Poblete, D., Mella-García, C., Cárcamo-Oyarzun, J., Cristi-Montero, C., RodríguezRodríguez, F., Delgado-Floody, P.,Von Oetinger,A., BalboaCastillo, T., Peña, S., Cuadrado, C., Bedregal, P., CelisMorales, C., García-Hermoso, A., \& Cortínez-O’Ryan, A. (2020). Boletín Chileno de Actividad Física Infantil y Adolescente 2018: Informe completo y comparaciones internacionales. Human Kinetics Journal, 17(8), 807-815. https: / / doi.org/10.1123/jpah.2020-0120

Aguayo Rousell, H. B. (Agosto de 2010). Las prácticas escolares de los educadores físicos. (IISUE-UNAM, Ed.) Perfiles Educativos, vol. XXXII(128), 97-117. Obtenido de http://www.scielo.org.mx/pdf/peredu/v32n128/ v32n128a6.pdf

Alacid , F., Vaquero, R., Lopez, P., \& Muyor, J. (2013). Imagen Corporal; revisión Bibliográfica. Nutrición Hospitalaria, 28 $\left(\mathrm{N}^{\circ} 1\right)$, 27-35. doi:dx.doi.org/10.3305/ nh.2013.28.1.6016

Asamblea Médica Mundial. (2008). Declaración de Helsinki de la Asociación Médica Mundial. Principios éticos para las investigaciones médicas en seres humanos. Corea: $59^{a}$ Asamblea General, Seúl, Corea, octubre 2008.

Asociación Médica Mundial. (22 de Julio de 2015). Asociación Médica Mundial. Obtenido de WMA: http:// www.wma.net/es/30publications/10policies/b3/

Argibay, J. C. (2006). Técnicas psicométricas, cuestiones de confiabilidad y validez . Subjetividad y procesos cognitivos, (15) 33. http://dspace.uces.edu.ar:8180/jspui/handle/ $123456789 / 765$

Astigarraga, E. (2003). El método Delphi. San Sebastia: Universidad de Deusto. http: / / www.academia.edu/1778723/ El_m\%C3\%A9todo_delphi

Baile, J., Garrido, E., \& Guillen, F. (Diciembre de 2002). Insatisfacción corporal en adolescentes medida con el Body
Shape Questionnaire (BSQ): efecto del anonimato, el sexo y la edad. Revista Internacional de psicología clínica y de la Salud, 2(3), 439-450. Obtenido de https:// www.researchgate.net/publication /

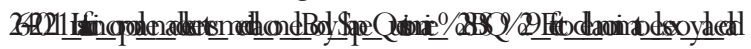

Behar, R. (2010). La construcción cultural del cuerpo: El paradigma de los trastornos de la conducta alimentaria. Revista chilena de neuro-psiquiatría, 319-334.

Blanco Ornelas, L., Tristán Rodríguez, J., Mayorga-Vega, D. \& Ornelas Contreras, M. (2016). Insatisfacción corporal en universitarios mexicanos deportistas y no deportistas. Retos, 30, 168-170. https://doi.org/10.47197/ retos.v0i30.50216

Buss, M., López, M., Rutz,A., Coelho, S., Oliveira, I., \& Mikla, M. (2013). Grupo Focal: una técnica de recogida de datos en investigaciones cualitativas. Index de enfermería, 75-78.

Caballero Huarcaya , L. (2008). Relación entre la autopercepción de la imagen corporal y hábitos de alimentación en adolescentes del 5 to año de secundaria de la Institución Educativa Teresa Gonzáles de Fanning, 2007. Lima: Universidad Nacional Mayor De San Marcos.

Cádiz Chacón, P., Barrio Mateu, L., León Valadares, D., Hernández Sánchez, Á., Milla Palma, M. \& Sotomayor Fernández, M. (2021). Motivación contextual desde la autodeterminación en las clases de Educación Física. Retos, 41, 88-94. https://doi.org/10.47197/ retos.v0i41.80998

Cárcamo Oyarzún, J. (2012). El profesor de educación física desde la perspectiva de los escolares. Estudios Pedagógicos, 105-119. Recuperado el 2015 de Diciembre de 11, de http: / / www.scielo.cl/pdf/estped/v38n1/art06.pdf

Casillas-Estrella, M., Montaño-Castrejón, N., ReyesVelázquez, V., Bacardí-Gascón, M., \& Jimenez-Cruz, A. (Diciembre de 2006). A mayor IMC mayor grado de insatisfacción de la imagen corporal. Revista Biomédica, 17(4), 243-249. Recuperado el 03 de Diciembre de 2015, de http: / /www.medigraphic.com/pdfs/revbio/bio2006/bio064b.pdf

Cea Morales, S., Véliz Véliz, C., Aravena Garrido, C., \& Maureira Cid, F. (Enero de 2013). Percepción de los estudiantes de educación física de la UISEK de Chile hacia sus profesores. (S. d. Murcia, Ed.) Cuadernos de Psicología del Deporte, 23-28. Recuperado el 2015 de 12 de 03, de http: / / revistas.um.es/cpd/article/view/191051/ 157771

Ceballos-Gurrola, O., Medina-Rodríguez, R., Juvera-Portilla, J., Peche-Alejandro, P., Aguirre-López, L. \& RodrígeuzRodríguez, J. (2020). Imagen corporal y práctica de actividades físico-deportivas en estudiantes de nivel secundaria. Cuadernos de Psicología del Deporte, 20(1), 252-260. Recuperado de https://scielo.isciii.es/ scielo.php?script $=$ sci_arttext\&pid $=$ S 1578 84232020000100019

Colunga-Rodríguez, B., Ángel-González, M., Vázquez-Juárez, 
C., Vázquez-Colunga, J., Castellanos-Ángeles, R., Colunga-Rodríguez, C. \& Davalos Picazo, G. (2020). Motivos para la Práctica del Ejercicio Físico en Estudiantes de Secundaria. Retos, 38, 89-94. https://doi.org/ 10.47197/retos.v38i38.71569

Contreras Jordán, O., Gil Madrona, P., García López, L., Fernández Bustos, J. \& Pastor Vicedo, J. (2012). Incidencia de un programa de Educación Física en la percepción de la propia imagen corporal, Revista de Educación, 357, 281-303, https: / / doi.org/10-4438/1988-592X-RE2010-357-061

Cordeiro Beling, M. T., Ribeiro Ferreira, M. F., Miranda de Araujo, A. M., Silveira Barros, A. F., Beling, G., \& Alves Lamounier, J. (2012). Alterações na imagem corporal entre adolescentes do sexo feminino e fatores associados. Revista oficial do núcleo de estudos da saúde do adolescente, 9(4), 11-18.

Cuadra Martinez, D., Georgudis Mendoza, C., \& Alfaro Rivera, R. (2012). Representación social de deporte y educación física en estudiantes con obesidad. Revista Latinoamericana de Ciencias Sociales, Niñez y Juventud, 983-1001.

Da Silva, L., Rabelo, A., Menezes, R., \& Knackfuss, M. (2008). Imagen corporal, Actividad Física y Factores de Riesgo. Revista de salud pública, 550-560.

Do Santos Silva, M., \& Leal Cortéz, A. (2017). Nivel de actividad física y la percepción de la imagen corporal de estudiantes - una revisión sistemática, Revista Ciencias de la Actividad Física, 18(1), 61-72. Recuperado de https:// www.redalyc.org/journal/5256/525664809006/ html/

Duncan, M., Al-Nakeeb, Y., Nevill, A., \& Jones, M. (Octube de 2004). Body image and physical activity in British secondary school children. European Physical Education Review, 10, 243-260. doi:10.1177/1356336X04047125

Durán, S., Rodriguez, M., Record, J., Barra, R., Olivares, R., Tapia,A., ... Neira, A. (2013). Autopercepción de la imagen corporal en estudiantes universitarios de Chile y $\mathrm{Pa}$ namá. Revista chilena de nutrición , 26-32.

Escobar-Pérez, y Cuervo-Martínez, A. (2008). Validez de contenido y juicio Experto. Una aproximación a su utilización. Avances en Medición, 6, 37-36. Recuperado de https: / /dialnet.unirioja.es/servlet/articulo?codigo $=2981181$

Estévez Díaz, M. (2012). Relación entre la insatisfacción con la imagen corporal, autoestima, autoconcepto físico y la composición corporal en el alumnado de segundo ciclo de educación secundaria de la ciudad de Alicante. Granada: Universidad de Granada.

Fernandez, J., Contreras, O., García, L., \& Gonzalez, S. (2010). Autoconcepto físico según la actividad físicodeportiva realizada y la motivación hacia ésta. Revista latinoamericana de psicología, 251-263.

Finato, S., Rech, R. R., Migon, P., Gavineski, I. C., deToni,V., \& Halpem, R. (2013). Body image insatisfaction in students from the sixth grade of public schools in Caxias do Sul,
Southern Brazil. Revista Paulista de pediatría, 65-70.

Flick, U. (2004). Introducción a la investigación cualitativa. Madrid: Morata. Recuperado de http:// investigacionsocial. sociales.uba.ar/wp-content/ u p loads/s i t e s/ $103 / 2013 / 03 /$ INVESTIGACIONCUALITATIVAFLICK.pdf

Fuentes Vilugrón, G., \& Lagos Hernández, R. (2019). Motivaciones hacia la práctica de actividad física-deportiva en estudiantes de La Araucanía. Revista Ciencias de la Actividad Física UCM, 20(2). https://doi.org/10.29035/ rcaf.20.2.3

Fuentes-Vilugrón, G., \& Lagos-Hernández, R. (2020). Razones para la no práctica física y deportiva en adolescentes de la Región de Araucanía, Chile. Pensar en Movimiento: Revista de ciencias del ejercicio y la salud, 18(2), 99-113. http: / /dx.doi.org/10.15517/pensarmov.v18i2.40531

Galán, I., Boix, R., Medrano, M., Ramos, P., Rivera, F., \& Moreno, C. (2014). Individual Factors and School-Based Policies Related to Adherence to Physical Activity Recommendations in Spanish Adolescents. Prevention Science, 15, 588-599. https://doi.org/10.1007/s11121013-0407-5

George Reyes, C., \& Trujillo Liña, L. (2018). Aplicación del Método Delphi Modificado para laValidación de un Cuestionario de Incorporación de las TIC en la Práctica Docente. Revista Iberoamericana de Evaluación Educativa, 11(1), 113-135. doi:https://doi.org/10.15366/ riee2018.11.1.007

Gobierno de Chile. (2013). EligeVivir Sano. Recuperado el 04 de Octubre de 2015, de http:/ / eligevivirsano.gob.cl

Gobierno de Chile. (06 de Marzo de 2015). Ministerio del desarrollo Social. Recuperado el 04 de Octubre de 2015, de http:/ /www.ministeriodesarrollosocial.gob.cl/noticias/2015/03/06/ministra-villegas:-\%E2\%80\%9Cnopuede-haber-educacion-de-calidad,-si-no-hay-vida-saludable-en-las-escuelas $\%$ E2\%80\%9D

Gómez, M. (2013). Relación entre la autopercepción de la imagen corporal y las clases de educación física, segun su nivel de intensidad y diversión, en alumnos de educación secundaria. Motricidad. European journal of human movement, 99-109.

Hernández Sampieri, R., Fernández Collado, Carlos., \& Baptista Lucio, P. (2014). Metodología de la Investigación. México: McGRAW-HILL. Recuperado de http: / / observatorio.epacartagena.gov.co/wp-content/ uploads/2017/08/metodologia-de-la-investigacionsexta-edicion.compressed.pdf

Jansen, P., Zayed, K., \& Kittsteiner, J. (2021). Body image and the relation to mindfulness and self-compassion in physical education students: a cross-cultural study. Health psychology research, 8(3), 9172. https://doi.org/10.4081/ hpr.2020.9172

Lagos Hernández, R. (2010). Medición y comparación a través del Body Shape Questionnaire de la imagen corporal 
de niños y niñas de 11 a 14 años de tres comunas de la Región de la Araucanía. Revista Ciencias de la actividad física de la Universidad Católica de Maule, 23-28.

Lagos, H. R. (Julio de 2011). La motricidad nuestra de cada día. Análisis de los alcances conceptuales de la Motricidad Humana como ciencia emergente. Revista Motricidad $\mathrm{Hu}$ mana, 12(1), 51-57. Recuperado el 2015 de 12 de 9, de http:// dialnet.unirioja.es / servlet/ articulo?codigo $=3706735$

Lizana Arce, P., Almagiá Flores, A., Simpson Lelievre, M., Binvignat Gutierrez, O., Ivanovic Marincovich, D., \& Berral de la Rosa, F. (2011). Aproximación a la tendencia secular del estado nutricional y composición corporal en escolares de enseñanza secundaria,V región, Chile: 1985-2010. International Journal of morphology, 473-478.

López Pastor,V. (2012). Didáctica de la Educación Física, desigualdad y transformación social. Estudios pedagógicos(1), 155-176.

Malina, Robert. (2012). Adherence to Physical Activity From Childhood to Adulthood: A Perspective From Tracking Studies. Quest, 53(3), 346-355. https://doi.org/ 10.1080/00336297.2001.10491751

Ministerio de Educación. (2013). Curriculum en linea. Recuperado el 05 de Octubre de 2015, de http:// www.curriculumenlineamineduc.cl/605/articles21314_programa.pdf

Miranda, V., Conti, M., Henrique, P., Rocha, R., \& Ferreira, M. (2014). Imagen corporal en distintos periodos de la adolescencia. Revista Paulista de pediatría, 63-69. doi:dx.doi.org/10.1590/S0103-05822014000100011.

Moreno Doña,A., Gamboa Jimenez, R., \& Poblete Galvez, C. (Abrl - Junio de 2014). La Educación Física en Chile: Análisis crítico desde la documentación Ministerial. Revista Brasileña de Ciencias del Deporte, 411-427. Obtenido de http: / / www.scielo.br/pdf/rbce/v36n2/01013289-rbce-36-02-00411.pdf

Moreno Murcia, J. A., \& Cervelló, E. (2003). Pensamiento del alumnado hacia la Educación Física: Su relación con la práctica deportiva y el caracter del educador. (E. U. Salamanca, Ed.) Enseñanza(21), 345-362. Recuperado el 2015 de Diciembre de 10, de http://rca.usal.es/ index.php/0212-5374/article/view/4068/4092

Moreno, J., \& Cervelló, E. (2003). Pensamiento del alumno hacia la educación física: Su relación con la práctica deportiva y el carácter del educador. Revista Enseñanza:Anuario interuniversitario de Didáctica, 345-362.

Moreno, M., \& Ortiz, G. (2009). Trastorno Alimentario y su Relación con la Imagen Corporal. Terapia Psicológica, 182$189 . \quad$ doi:dx.doi.org/10.4067/S071848082009000200004

Newman, K., Harrison, L., Dashiff, C., \& Davies, S. (2008). Relaciones entre los tipos de padres y comportamientos de riesgo en la salud del adolescente: Una revisión bibliográfica integrada. Revista latinoamericana de enfermagem,
142-150.

Nichols, S., Dookeran, S., Ragbir, K., \& Dalrymple, N. (2009). Body image perception and the risk of unhealthy behaviours among University students. West Indian Medical Journal, 58(5), 465-471.

Onofre, R., Gil, P., García, L., Fernandez, J., \& Pastor, J. (2009). Incidencia de un programa de educación física en la percepción de la propia imagen corporal. Revista de Educación, 281-303.

Onwuegbuzie, A., Leech, N., Dickinson, W., \& Zoran, A. (2011). Un marco cualitativo para la recolección y analisis de datos en la investigación basada en grupos focales. Paradigmas, 127-157.

Ornelas Contreras, M., Solís, O., Solano Pinto, N. \& Rodríguez-Villalobos, J. (2020). Imagen corporal percibida en mujeres adolescentes deportistas y no deportistas. Retos, 37, 266-237. https: / / doi.org/10.47197/ retos.v37i37.72386

Osses, S., \& Jaramillo, S. (2008). Metacognición: un camino para aprender a aprender. Estudios Pedagógicos, 34(1), 187 $197 . \quad$ doi:dx.doi.org/10.4067/S071807052008000100011

Perez Lopez, I., \& Delgado Fernandez, M. (2009). Efectos de un juego de rol sobre los procedimientos de práctica de actividad física relacionada con la salud en secundaria. Revista de Curriculum y formación del profesorado, 318-328.

Pérez, S., \& Romero, G. (2010). Imagen corporal en mujeres de tres zonas rurales de México: percepción y deseo. Salud pública de Mexico, 52(2), 111-118.

Perpiñá, C., \& Baños, R. (2007). Distorsión de la imagen corporal: un estudio en adolescentes. Cuadernos de Psicopedagogía, 6(11). Recuperado el 2015 de diciembre de 12, de http://pepsic.bvsalud.org/ s c i e l o.p p p ? p i d = S 1676 $10492007000100004 \&$ script $=$ sci_arttext

PeñaTroncoso, Sebastián, Espinoza Sánchez, Laura, Hernández Mosqueira, Claudio, Toro Arévalo, Sergio, Cárcamo Oyarzun, Jaime, Pérez Adasme, Gustavo, \& Velásquez González, Hugo (2021). Motives of Students for or against the Practice of Physical Exercise and How They Are Related with the Climate in Physical Education Classes. International Journal of environmental Research and Public Health, 18(16). https://doi.org/10.3390/ ijerph18168348

Pieron, M. (2007). Factores determinantes en la inactividad físico-deportiva en jóvenes y adolescentes. En V. C. Escolar (Ed.), Factores determinantes en la inactividad físico-deportiva en jóvenes y adolescentes, (págs. 15-67). Badajoz. Recuperado el 03 de Diciembre de 2015, de http:// www.plazadedeportes.com/imgnoticias/12456.pdf

Pilsa Doméne, C., Gutiérrez San Martín, M., \& Torres Benet, E. (Julio de 2007). Perfil de la educación física y sus profesores desde el punto de vista de los alumnos. (U. d. Valencia, Ed.) International Journal of Sport Science, III, 39- 
52. doi:10.5232/ricyde2007.00804

Plana Galindo, C. (1992). El Rol del Profesor de Educación Física. Revista Apunts Educación Física I Esports, 58-63.

Poblete Galvez, C., Moreno Doña, A., \& Rivera García, E. (2014). Educación Física en Chile: Una historia de la disciplina en los escritos de la primera publicación oficial del Instituto de Educación Física de la Universidad de Chile (1934-1962). Estudios Pedagógicos, 40(2), 265-282.

Ramos, P., Rivera, F., \& Moreno, C. (2010). Diferencias de sexo en imagen corporal, control de peso e Índice. Psicothema, 78-83.

Requena Gonzalez, S. (2012). Pesquisas y criterios sobre la adolescencia. Revista de psicológica, 9-35.

Reyes, D., Delgado-Floody, P., \& Martínez-Salazar, C. (2020). Hábitos alimentarios, imagen corporal y condición física en escolares chilenos según niveles de actividad física y estatus corporal. Nutrición Hospitalaria, 37(3), 443-449. https: / / dx.doi.org/10.20960/nh.02795

Rodríguez, A., Goñi, A., \& Ruiz de Azúa, S. (2006). Autoconcepto físico y estilos de vida en la adolescencia. (U. d. Vasco, Ed.) Intervención psicosocial, 15(1), 81-94. Obtenido de http://scielo.isciii.es/pdf/inter/v15n1/ v15n1a06.pdf

Rodriguez, L., \& Pizarro, T. (2006). Situación nutricional del escolar y adolescente en Chile. Revista chilena de pediatría, 70-80.

Ruiz Bueno, A. (01 de Abril de 2014). Dipòsit Digital de la Universitat de Barcelona. Recuperado el 28 de Agosto de 2021, de http://diposit.ub.edu/dspace/handle/2445/ 53152

Sabatini, F. (2006). El Interés por la Educación Física. Universidad Abierta Interaméricana. Buenos Aires: Licenciatura en Educación Física. Recuperado el Diciembre de 2015, de http://imgbiblio.vaneduc.edu.ar/fulltext/files/ TC072119.pdf

Salinas, J., \& Vio, F. (Octubre de 2002). Promoción de la Salud en Chile. Revista chilena de Nutrición, 29(1), 164173. dx.doi.org/10.4067/S0717-75182002029100001

Salvador Soler, N., Lagos Hernández, R., Grupos de Profesores de Educación Física, \& Valdés Badilla, P. (2018). Relación de la imagen corporal con la ingesta de grasas y micronutrientes en escolares chilenos: un estudio descriptivo correlacional, Nutrición Clínica y Dietética Hospitalaria, 38(1), 40-45. dx.doi.org/10.12873/ 381NSalvador

Serón , P., Muñoz , S., \& Lanas , F. (2010). Nivel de actividad física medida a través del cuestionario internacional de actividad física en población Chilena. Revista médica de Chile, 138, 1232-1239. Recuperado el 2015 de diciembre de 09, de http://www.scielo.cl/pdf/rmc/ v138n10/art\%2004.pdf

Solana, A. (2003). La participación de los alumnos en las clases de educación física: una perspectiva cualitativa de sus comportamientos. Revista digital de Buenos Aires,
19(67). Obtenido de http://www.efdeportes.com/ efd67/particip.htm

Soto,A., Cáceres, K., Faure, M., Gásquez, M., \& Marengo , L. (2013). Insatisfacción corporal, busqueda de la delgadez y malnutrición por exceso, un estudio descriptivo correlacional en la población de estudiantes de 13 a 16 años de la ciudad de Valparaiso. Revista chilena de nutrición, 10-15.

Strauss, A., \& Corbin, J. (2002). Bases de la investigación cualitativa.Técnicas y procedimientos para desarrollar la teoría fundamentada. Medellín: Editorial Universidad de Antioquia. Obtenido de https:// diversidadlocal.files.wordpress.com/2012/09/basesinvestigacion-cualitativa.pdf

Stuart Rivero, A., Lopez Gutierrez, C., \& Granado Mejías, A. (2012). Conocimientos formativos básicos para una Educación Física saludable. Estudios Pedagógicos, 197-214.

Torre, R. E. (1998). La actividad físico-deportiva extraescolar y su interrelación con el área de educación física en el alumnado de enseñanzas medias. Granada: Repositorio Institucional de la Universidad de Granada.

Trejo Ortiz, P., Castro Veloz, D., Facio Solís, A., Mollinedo Montano, F., \& Valdez Esparza, G. (2010). Insatisfacción con la imagen corporal asociada al indice de masa corporal en adolescentes. Revista cubana de enfermería, 150-160.

Urrutia, S.,Azpillaga, I., \& Gurutze, L. (2010). Relación entre la percepción de estado de salud con la práctica fisicodeportiva y la imagen corporal en adolescentes. (F. d. Mucia, Ed.) Cuadernos de psicología del deporte, 10, 51 56. Recuperado el 3 de Diciembre de 2015, de https: / / digitum.um.es/xmlui/handle/10201/35284

Valdez Medina, J. L., \& Guadarrama Guadarrama, R. (2008). Imagen coporal en bailarinas de Ballet y estudiantes de Bachillerato. Psicología y Salud, 18(001), 63-68. Recuperado el 2015 de diciembre de 11, de http:// www.redalyc.org/pdf/291/29118107.pdf

Vaquero, R., Alacid, F., Muyor, J., \& López, P. (2013). Imagen corporal; revisión bibliográfica. Nutrición Hospitalaria, 27 35.

Woodburn, S., Boschini, C. , \& Fernández, H. (1997). La imagen corporal en niños. Costa Rica: Universidad de Costa Rica.

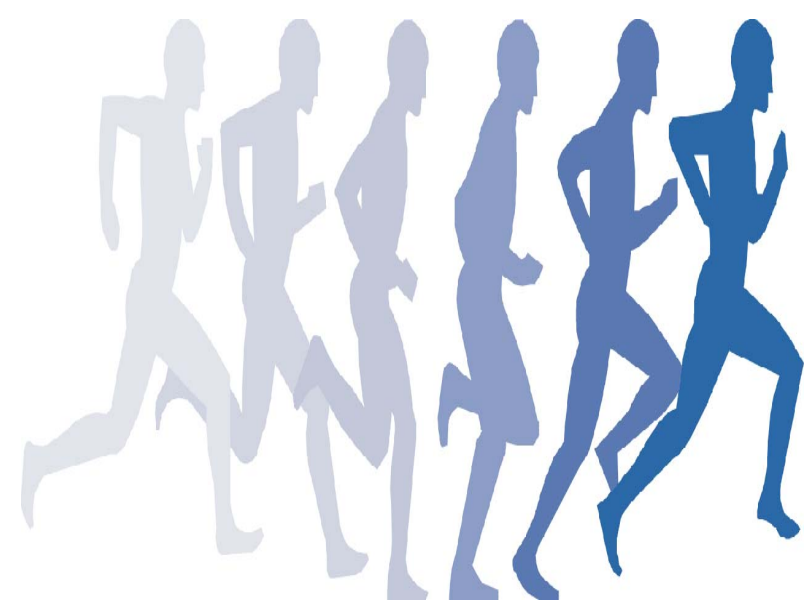

